\title{
Desarrollo de un sistema de votación electrónico con mecanismo biométrico de autentificación.
}

\section{Development of an electronic voting system with biometric authentication mechanism.}

Ing. Richard Alejandro Macías Lara. ${ }^{1}$, Ing. Adrián Oswaldo Mendoza Antón. ${ }^{2}$, MSc. Jorge Luis Puyol Cortez. ${ }^{3} \&$ MSc. Ramiro Enrique Guamán Chávez. ${ }^{4}$

Recibido: 02-09-2017 / Revisado:03-11-2017 Aceptado: 10-12-2017/ Publicado: 01-01-2018

\begin{abstract}
.
DOI: $\underline{\text { https://doi.org/10.33262/cienciadigital.v2i1.17 }}$
\end{abstract}

For the fulfillment of this project begins with a deep investigation in the different media, such as the internet and bibliographic material, in what refers to biometric technology issues, which is carefully analyzed to define what type of biometric system is the more convenient to use for the web application.

It should be noted that a field and documentary research is done with a mixed approach with a sample of 144 students, a table of impacts was made to evaluate the system in order to determine the advantages and usability of the same in the student elections, the table it consists of qualifying on the scale of 0-3 with equivalence of no impact at high positive impact.

The system will show pictures of the members of each participant list to the Cogovernment in the application's home screen, their names and their proposal; There is also a tab that shows the information regarding the participants to School Councils. It is concluded that the application has a positive average impact in that it speeds up the entire election process and shows results at the end of the elections, avoiding all the possible errors that are committed with the conventional system, generating a degree of satisfaction for the students.

Keywords: Elections, Software, Biometrics, Automation, Computer Application

\footnotetext{
${ }^{1}$ Universidad Técnica Luis Vargas Torres de Esmeraldas Ext. La Concordia, Esmeraldas, Ecuador alejandro.macias.new@gmail.com

${ }^{2}$ Universidad Técnica Luis Vargas Torres de Esmeraldas Ext. la Concordia, Oswaldo.mendoza@utelvt.edu.ec

${ }^{3}$ Universidad Técnica Luis Vargas Torres de Esmeraldas Ext. la Concordia, jorge.puyol@utelvt.edu.ec

${ }^{4}$ Universidad Técnica Luis Vargas Torres de Esmeraldas Ext. la Concordia, guaman@utelvt.edu.ec
} 


\section{Resumen.}

Para el cumplimiento de este proyecto se comienza con una investigación profunda en los diferentes medios, como son el internet y material bibliográfico, en lo que se refiere a temas de tecnología biométrica, el cual se analiza cuidadosamente para definir qué tipo de sistema biométrico es el más conveniente de usar para la aplicación web.

Cabe recalcar que se hace a una investigación de campo y documental con un enfoque mixto con una muestra de 144 estudiantes, se realizó una tabla de impactos para evaluar el sistema con el fin de determinar las ventajas y usabilidad del mismo en las elecciones estudiantiles, la tabla consiste calificar en la escala de -3 a 3 , acorde al impacto que va generado el sistema.

El sistema va a mostrar fotos de los integrantes de cada lista participante al Cogobierno en la pantalla de inicio de la aplicación, sus nombres y la propuesta de los mismos; también existe una pestaña que muestra la información referente a los participantes a Consejos de Escuela. Se concluye que la aplicación tiene un impacto medio positivo por cuanto agilita todo el proceso de elecciones y muestra resultados al término de las elecciones evitando todos los posibles errores que se cometen con el sistema convencional generando un grado de satisfacción a los estudiantes.

Palabras Claves: elecciones, software, biometría, automatización, aplicación informática.

\section{Introducción.}

Se entenderá como voto electrónico a aquel que se realiza por medio de algún dispositivo electrónico en forma automática en una urna electrónica o una PC (Compuador Personal). En aquel terminal se presentan todas las opciones en competencia (partidos políticos o candidatos) para permitir la selección inmediata. De igual manera, el escrutinio de votos es inmediato lo que posibilita que se entreguen resultados en corto tiempo (Tuesta Soldevilla, 2004).

La aplicación de nuevas tecnologías en materia electoral ha aumentado a nivel mundial en los últimos años. El voto electrónico es un término que se ha empleado en forma general para identificar este proceso. De manera general existen dos tipos de voto electrónico. El primero, se encuentra físicamente supervisado por representantes del órgano electoral y el segundo es el remoto, en el cual el votante no necesita desplazarse a una casilla electoral y se realiza mediante la tecnología de telefonía celular o por internet (Zissis \& Lekkas, 2011)

El voto electrónico tiene diversos argumentos a favor y en contra. Los primeros son económicos, ambientales y políticos, entre los cuales destacan la reducción del costo de las 
elecciones, la protección del medio ambiente, la agilización del cómputo de sufragios, lo que supone un ahorro financiero importante, evita desperdicio de materiales, cuya producción depreda el ambiente y su eliminación lo contamina, y reduce la incertidumbre de los resultados, (Velázquez Trujillo, 2010)

Según Tuesta Soldevilla (2004). El proceso de elecciones por votación democrática para la determinación de dignidades, se lleva a cabo en muchas instituciones estudiantiles, este proceso puede llegar a tomarse complejo y tedioso. Por tanto, se hace necesario responder a estas exigencias para que todo este proceso que se realiza se agilice y automatice. Uno de los principales problemas que existe en la PUCESE es la dificultad del conteo manual de los votos y esto genera información poco confiable, así como retrasos en la generación de reportes y en consecuencia insatisfacción en los estudiantes al no tener bases que justifiquen los resultados expuestos.

Los estudiantes por lo general nunca se enteran que tienen que votar por que existe una gran brecha de información, de parte de la universidad hacia los estudiantes. Hay alumnos que arrastran materias y no asisten algunos días a la semana, coincidiendo estos días con las votaciones, provocando que estos se queden sin votar. La universidad no cuenta con un servicio de votación electrónica que genere un envió automático vía email a estudiantes recordando que se acercan las votaciones, que ofrezca seguridad y satisfacción a los estudiantes al mostrar los resultados del ganador inmediatamente después de las votaciones y cada lista con sus respectiva suma de votos. (Díaz, 2008)

Se puede presentar errores al momento de generar reportes de los estudiantes que no han votado, esto conlleva a la sanción errónea de algún estudiante que haya votado y se encuentre en la lista o de estudiantes que no hayan votado y no estén en la lista. Mediante el presente trabajo se determina que no basta con llevar una buena organización en el proceso electoral estudiantil, puesto que los estudiantes se cansan al pasar todo el día registrando los votos y al término del mismo, comenzar a realizar los escrutinios como pasa en la Pontificia Universidad Católica del Ecuador Sede en Esmeraldas (PUCESE), por lo que se requiere de la intervención tecnológica y se ha establecido que la biometría es la mejor opción, para que se mejore y agilice el proceso electoral, ya que al ser manual resulta ser un proceso tedioso para los responsables del mismo. Las elecciones para federación de estudiantes en la Pontificia Universidad Católica del Ecuador Sede Esmeraldas (PUCESE), se realizan en el primer periodo de cada año académico, con un sistema manual tradicional, mas no se ha llevado el proceso electoral a un sistema electrónico como el que se propone.

Este sistema manual, trae consigo inconvenientes tales como: impresión y clasificación de papeletas, poca fiabilidad a la hora del escrutinio, errores en generación de listados de votantes, etc., situación que ha llevado a pensar en utilizar tecnología informática de punta, 
que permita automatizar y modernizar el proceso electoral para que esté acorde al desarrollo del mundo actual. El diagnóstico que se realizó a los estudiantes de la PUCESE, respalda la idea de desarrollar un sistema web para las elecciones estudiantiles con autentificación biométrica, que automatice este proceso y elimine todas las posibles incomodidades que se vienen generando con el sistema convencional, los mismos que fueron evidenciados en el estudio diagnóstico, como son: demora al mostrar resultados, confusión de papeletas, registro erróneo de estudiantes que no votaron, etc. La propuesta de automatizar el proceso de las elecciones, beneficiará en general a la comunidad universitaria de la PUCESE, ya que le permitirá contar con un servicio tecnológico que la resaltará de otras universidades. Por lo antes mencionado, se plantea el presente trabajo, cuyo objetivo general es el de generar información fiable y satisfacción a los estudiantes al mostrar resultados inmediatamente después de las votaciones de la PUCESE, mediante un sistema de votación electrónico con tecnología biométrica.

El proyecto se realiza con la finalidad de garantizar información fiable, proporcionándole a los participantes en este caso los estudiantes, resultados de cada lista inmediatamente después al termino de las elecciones, también se optimizará gran cantidad de tiempo y reducirá cualquier tipo de errores que se cometen al momento de los escrutinios, que se hacen manualmente y esto conlleva a veces a confusión de información. El beneficio no solamente será para los estudiantes, también se beneficiará la PUCESE en general al contar con un servicio web para elecciones que la resaltará de otras universidades, será más eficiente la manipulación de datos y la información será mucho más confiable, contará con un mecanismo que podrá comunicar oportunamente a los estudiantes vía email sobre la fecha en la que se realizará las inscripciones de candidaturas y elecciones.

Se tiene que considerar que los estudiantes deben registrar la huella dactilar para poder ejercer su derecho al voto, este proceso se realiza para nuevos estudiantes que ingresan a la PUCESE y los ya existentes, los que ya tienen registrado su huella no están obligados a volver a hacerlo; el Tribunal Electoral Estudiantil podrá generar reportes de los estudiantes que cumplen con requisitos para poder participar como candidatos, padrón electoral, listas ganadoras y listas inscritas, todo ello con la ayuda del generador de reportes. Cabe indicar que, para evitar duplicidad de datos, con los sistemas informáticos que posee la PUCESE, el sistema propuesto se acopla a la base de datos de la institución, mediante vistas, solo de las tablas relevantes para el funcionamiento de la propuesta.

\section{Métodos y materiales.}

La modalidad de la presente investigación es con un enfoque mixto cualitativo y cuantitativa , basándose en investigación de campo y documental, se pretende plantear una serie de alternativas que tiendan a solucionar este tipo de problemas, el cual se juegan intereses y 
necesidades que incluyen directamente en la implementación de un sistema de voto electrónico, se tomó una muestra de 144 estudiantes PUCESE, además se realizó una entrevista al presidente del tribunal electoral estudiantil sobre el proceso electoral, para el procesamiento de datos se utilizó Excel, formulas, tablas, gráficos para demostrar los resultado obtenido en esta investigación.

La técnica de la observación, internet, entrevista y encuesta aplicadas, profundiza para obtener los posibles problemas que se presentan e interpretar el grado de interés que tiene el gobierno PUCESE y el entrevistado sobre la propuesta de la aplicación web.

\section{Análisis Discusión de resultados}

El proyecto se realiza con la finalidad de garantizar información fiable, proporcionándole a los participantes en este caso los estudiantes, resultados de cada lista inmediatamente después al termino de las elecciones, también se optimizará gran cantidad de tiempo y reducirá cualquier tipo de errores que se cometen al momento de los escrutinios, que se hacen manualmente y esto conlleva a veces a confusión de información.

\section{Determinación de los requerimientos}

La tabla 1, muestra la descripción de los requerimientos, donde se visualizan los procesos que hasta la actualidad se venían ejecutando y lo que se propone automatizar.

TABLA 1: ReQUERIMIENTOS DEL PROCESO

\begin{tabular}{clcc}
\hline No & \multicolumn{1}{c}{ Proceso } & Actual & Propuesto \\
\hline 1 & Voto del estudiante & Manual & Automatizado \\
2 & Registro de listas & Manual & Automatizado \\
3 & Registro huella dactilar & No existe & Automatizado \\
4 & Reporte de ganadores & Manual & Automatizado \\
5 & Reporte de candidatos & No existe & Automatizado \\
6 & Reporte de propuestas & Manual & Automatizado \\
7 & Reporte de estudiantes que no sufragaron & Manual & Automatizado \\
8 & Reporte de escrutinio & Manual & Automatizado \\
9 & Reporte de Escuelas que no participaron & Manual & Automatizado \\
10 & Aviso masivo de elecciones a estudiantes & No existe & Automatizado \\
11 & Editar listas & Manual & Automatizado \\
12 & Registro de nuevo Tribunal Estudiantil & Manual & Automatizado \\
13 & Reporte de patrón electoral & Automatizado & Automatizado \\
\hline
\end{tabular}

ElABORAdO POR: GRUPO DE INVESTIGACIÓN 


\section{Esquema Funcional del sistema}

A través del sistema, se podrá acceder a la información de los candidatos, propuestas, resultados, padrón electoral desde cualquier parte del mundo ya sea mediante computadoras, tabletas o celulares, ya que es una aplicación web y a su vez responsiva (adaptativa a cualquier tipo de pantalla), para votar, solo se puede acceder desde una computadora disponible en las Juntas Receptoras de Voto (JRV) de la institución con su respectivo dispositivo biométrico y sólo tendrán acceso el día de las elecciones, en la figura 1, se puede ver un esquema de cómo funciona la aplicación web.

FIGURA 1: ESQUEMA FUNCIONAL DE LA APLICACIÓN

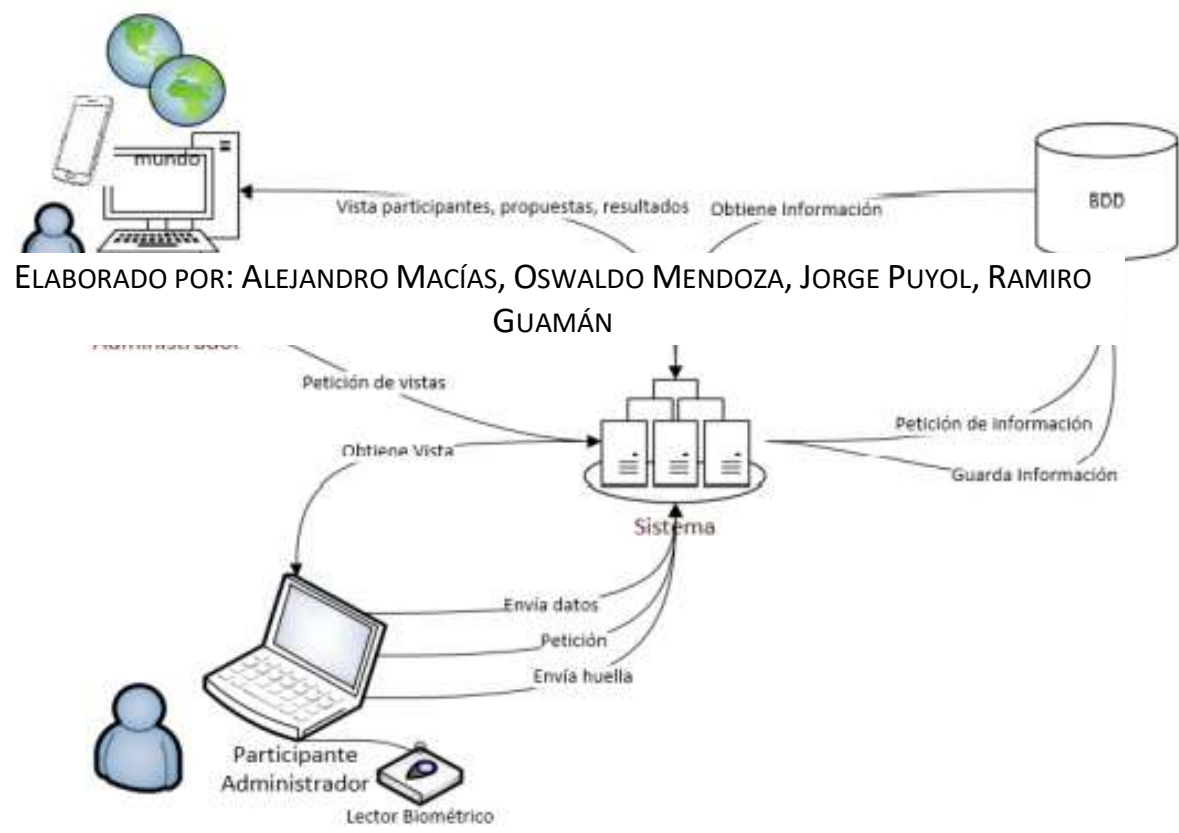

ElABORAdO POR: GRUPO DE INVESTIGACIÓN. 
FigurA 2: MODELO LÓGICO DE LA BDD

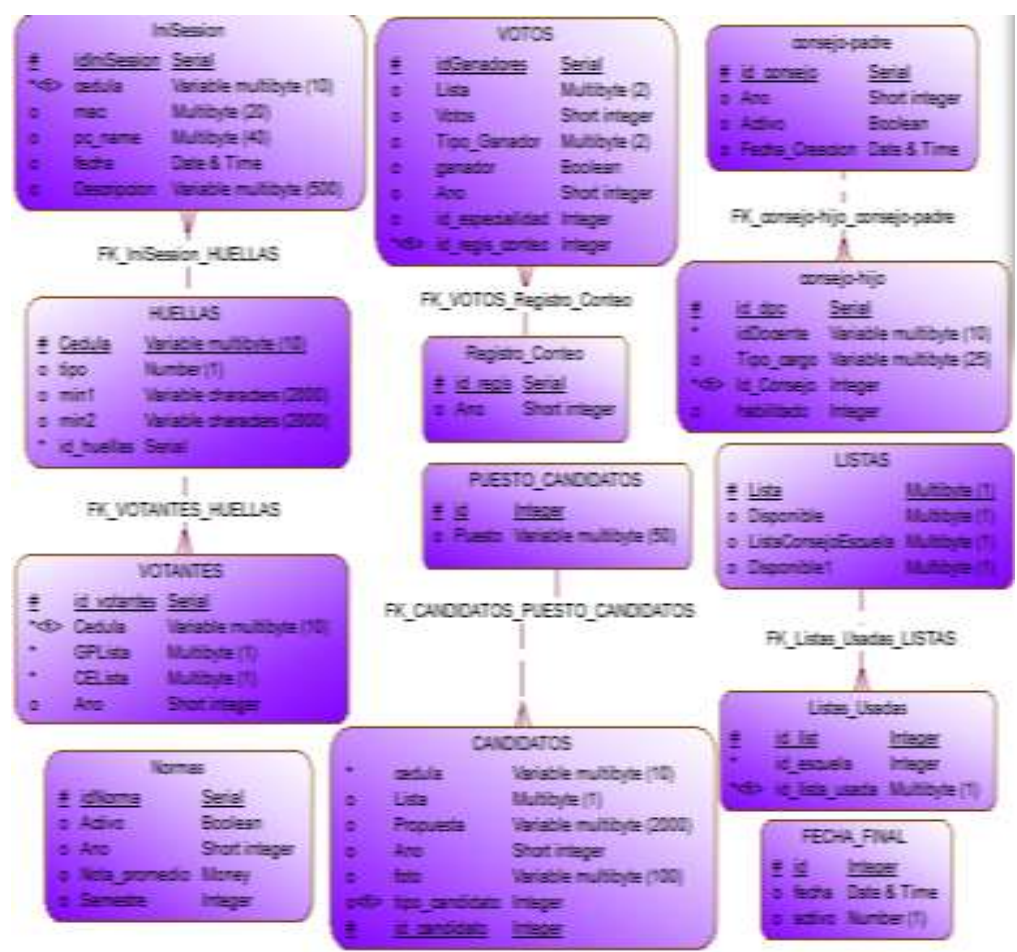

ELABORADO POR: GRUPO DE INVESTIGACIÓN.

FIGURA 3: MODELO CONCEPTUAL.

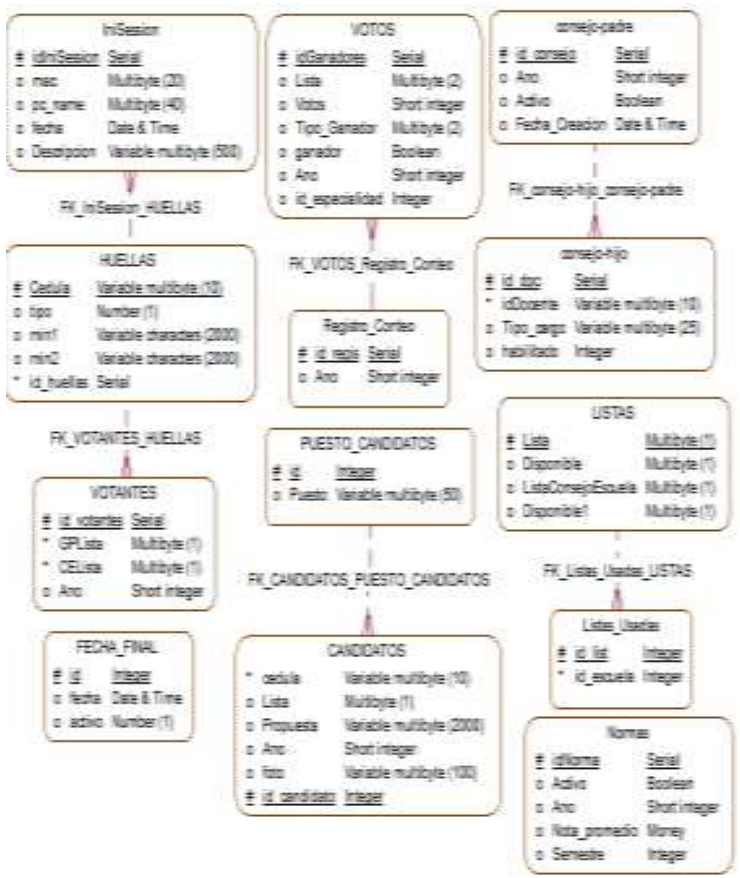

Elaborado POR: GRUPO DE INVESTIGACIÓN. 


\section{Desarrollo del sistema.}

\section{Determinación del lenguaje de programación.}

Se empleó a PHP como un lenguaje de programación de lado del servidor, de código libre, por su robustez y facilidad de código, es una de las muchas tecnologías que se pueden utilizar para afrontar un desarrollo web. (Cobo, Gomez, Perez, \& Rocha, 2005)

El código que se desarrolla es eficaz y a la vez entendible por lo que permite una fácil comunicación con las diferentes bases de datos; también se empleó JavaScript, Ajax, y JQuery con HTML5 para darle dinamismo y estilo al código desarrollado (Alvarez, 2010), a su vez con codificación responsive para que se adapte a dispositivos móviles.

\section{Determinación del sistema manejador de bases de datos.}

Se empleó el sistema manejador de bases de datos SQL SERVER 2012, siendo uno de los manejadores más utilizados en el mundo por diferentes organizaciones.

Por lo que también ha sido diseñado para trabajar en entornos híbridos, ya sea de manera local o en la nube. Las nuevas herramientas de SQL Server y de Microsoft Azure facilitan aún más la creación de soluciones de aplicación de revisiones, de recuperación ante desastres y las copias de seguridad con Microsoft Azure. Estas herramientas proporcionan un traslado sencillo a la nube de bases de datos de SQL Server locales, lo que permite a los clientes usar sus conocimientos actuales para aprovechar las ventajas de los centros de datos globales de Microsoft. (Microsoft SQL Server, 2015).

Otro punto por el que se empleó, es porque la universidad cuenta con Licencia del software y tiene la mayoría de su información administrada por este manejador de Base de datos, así el sistema de votaciones se acoplará sin problemas con la base de datos de la universidad.

\section{Sistema biométrico a utilizar.}

Para este proyecto se optó por elegir un hardware con una tecnología rápida y precisa para mejorar el rendimiento y reducir el tiempo de acceso al sistema. Una de las empresas líder en todo el mundo dedicada a la óptica de tecnología de reconocimiento es Secugen creadora del sensor biométrico que se seleccionó para el proyecto, que es el Hamster Plus, uno de sus lectores más popular y versátil en el mercado con Auto-On y Smart Capture. (Secugen Corporation, 2012).

Las principales características de Hamster Plus son las siguientes:

- Alto rendimiento, sensor óptico de huellas dactilares sin necesidad de mantenimiento 
- Conexión USB

- Fácil acceso a cualquier dedo

- Verificación rápida y precisa

- Bajo Presupuesto

- Sensores Preciso

Este dispositivo funciona bajo toda plataforma Windows, cuenta con dll y ocx programados en Microsoft Visual Basic .Net para la utilización en cualquier proyecto, para el funcionamiento correcto de este dispositivo se deben registrar las dll y ocx en el sistema para poder acceder al dispositivo y utilizarlo en el proyecto.

Figura 4: DisPositivo BIOMÉTRICO

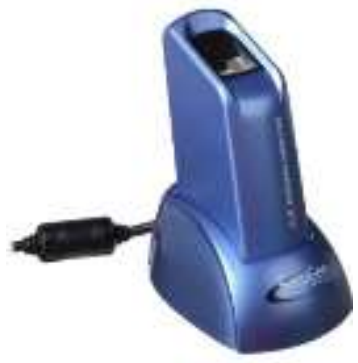

FUENTE: (SECUGEN CORPORATION, 2012).

ELABORADO POR: GRUPO DE INVESTIGACIÓN.

\section{Interfaz gráfica del sistema informático}

El sistema plantea interfaces gráficas dinámicas y versátiles, todo ello para la comodidad de los usuarios que empleen el sistema. A continuación, se detallan las interfaces gráficas principales del sistema:

FIGURA 5: INTERFAZ PRINCIPAL.

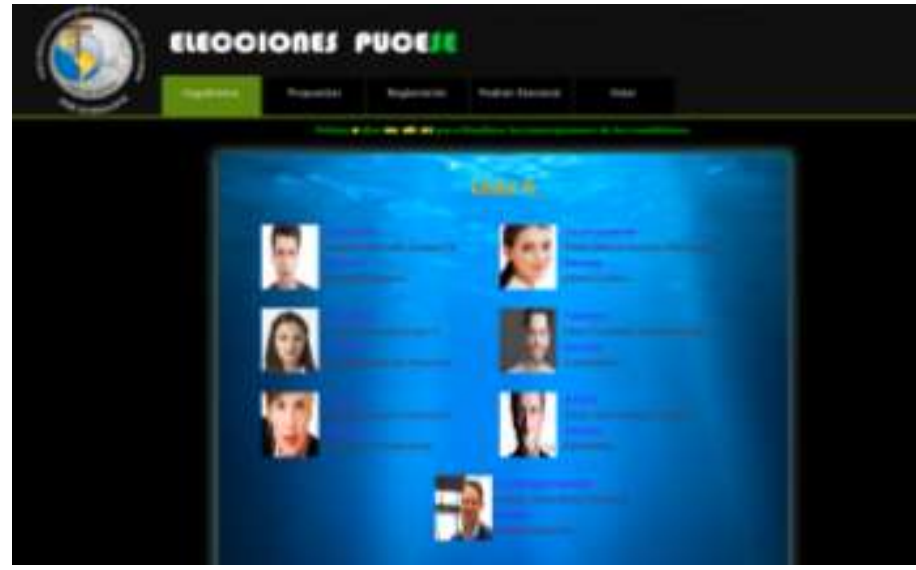

ELABORADO POR: GRUPO DE INVESTIGACIÓN. 
La Figura 5, muestra la interfaz para todos, en la que podrán ver: los participantes a Cogobierno con su respectiva foto, escuela y cargo que desempeñará, propuestas de cada lista y listas de consejos por cada escuela, el reglamento que rige a la PUCESE en las elecciones, el padrón electoral de los estudiantes que pueden votar.

El inicio de sesión que será mediante el uso del dispositivo biométrico y una pestaña de resultados que solo se habilitará el día de finalización de las elecciones, esta mostrará las listas ganadoras y un reporte de votos que obtuvieron todas las listas con la toda la información en cuando se refiere a las elecciones.

Figura 6: VOTACIONES.

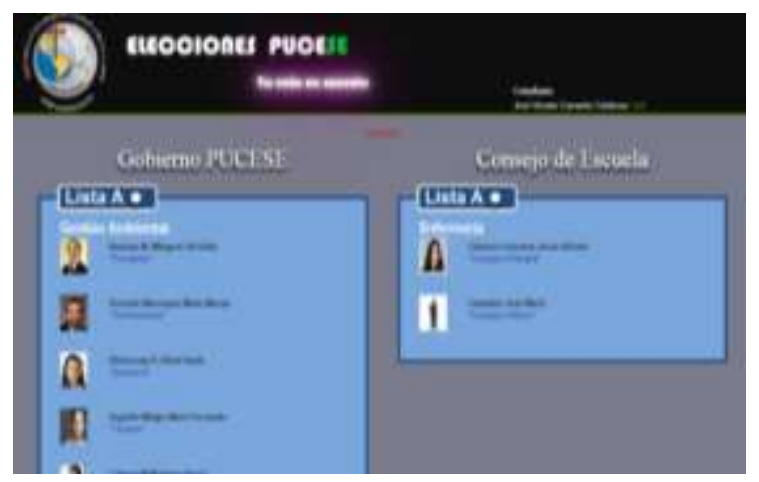

ELABORADO POR: GRUPO DE INVESTIGACIÓN.

La figura 6, muestra una ventana que sólo estará disponible el día de las elecciones para que los estudiantes registrados puedan ejercer el derecho al voto.

\section{Análisis e Impactos.}

Una vez culminado el desarrollo del sistema de votación electrónico para la PUCESE, se han determinado varios impactos en diferentes ámbitos como: Tecnológico, Ecológico, Económico y Administrativo. De la misma forma para poder interpretarlos, se utiliza una matriz base de impactos, la misma que aparece en la siguiente tabla:

Tabla 2: Matriz de impactos

\begin{tabular}{cl}
\hline & Matriz de Impactos \\
\hline-3 & Impacto Alto Negativo \\
-2 & Impacto Medio Negativo \\
-1 & Impacto Bajo Negativo \\
0 & No hay impacto \\
1 & Impacto Bajo Positivo \\
2 & Impacto Medio Positivo \\
3 & Impacto Alto Positivo \\
\hline
\end{tabular}

Fuente: Ángel, 2013, pág. 236 


\section{Impacto Tecnológico}

Tabla 3: Matriz impacto tecnológico

$\begin{array}{lllllllll}\text { Nivel de Impactos } & -3 & -2 & -1 & 0 & 1 & 2 & 3\end{array}$

\section{Indicador}

Automatización de procesos

Información rápida y oportuna

Manejo de Reportes

Total

\section{$\mathrm{X}$}

$\mathrm{X}$

$$
\mathrm{X}
$$

43

$$
\begin{gathered}
\text { Nivel de impacto tecnológico }=\frac{\sum}{\text { Número de Indicadores }} \\
\qquad \mathrm{NI}=\frac{7}{3}=2,3
\end{gathered}
$$

Nivel de Impacto tecnológico $=$ Medio positivo

ELABORAdO POR: GRUPO DE INVESTIGACIÓN.

El sistema de votación electrónico con mecanismo biométrico, logra informatizar uno de los procesos que hasta la presente fecha eran complejos y tedioso, por eso este indicador se valora como medio positivo.

Mediante el empleo de tecnología informática, la información será la que se requiera en el momento indicado, se pueden obtener resultados en cualquier momento, este indicador se valora como medio positivo.

Con la automatización de este proceso, se podrá obtener reportes de toda clase como: resultados de votos, proclamación de propuestas, estudiantes que no participaron, entre otros; por este motivo se ha valorado como alto positivo este impacto. 


\section{Impacto Ecológico}

Tabla 4: Matriz de Impacto Ecológico

$\begin{array}{llllllll}\text { Nivel de Impactos } & -3 & -2 & -1 & 0 & 1 & 2 & 3\end{array}$

\section{Indicador}

\section{Disminución de papelerías}

X

$\begin{array}{ccc}\text { Uso de herramientas tecnológica } & \mathrm{X} & \\ \text { Total } & -1 & 2\end{array}$

Nivel de impacto ecológico $=\frac{\sum}{\text { Número de Indicadores }}$

$$
\mathrm{NI}=\frac{1}{2}=0.5
$$

\section{Nivel de Impacto ecológico $=$ Impacto bajo positivo}

ELABORADO POR: GRUPO DE INVESTIGACIÓN.

Sin duda, gracias a la implementación del sistema, se disminuirá el uso de la papelería, por lo que todo será automático, por eso se ha valorado como medio positivo. Consume energía y cuando se desecha la tecnología, en la mayoría de los casos se hace inadecuadamente, y se ha evaluado como bajo negativo este indicador, debido a que la PUCESE ya cuenta con la tecnología computacional necesaria para el proyecto. 


\section{Impacto Administrativo.}

Tabla 5: Matriz de Impacto Administrativo.

$\begin{array}{llllllll}\text { Nivel de Impactos } & -3 & -2 & -1 & 0 & 1 & 2 & 3\end{array}$

Indicador

Proceso de elecciones.

$\mathrm{X}$

Administración de la Información $\quad X$

Total 4

Nivel de impacto Administrativo $=\frac{\sum}{\text { Número de Indicadores }}$

$$
\mathrm{NI}=\frac{4}{2}=2
$$

\section{Nivel de Impacto Administrativo = Impacto medio positivo}

ELABORADO POR: GRUPO DE INVESTIGACIÓN.

Con la ayuda del sistema de votación electrónico para la PUCESE, la administración constituida por el Tribunal Electoral Estudiantil tendrá procesos más eficientes, de forma que los procesos que solían darse manualmente, cambiarán significativamente, por eso se valora como medio positivo este indicador. La administración de la información es parte importante dentro de las votaciones, por lo que disminuirá recursos de tiempo y humanos, logrando un proceso eficiente y equilibrado, por eso se ha valorado como medio positivo. 


\section{Impacto Económico}

Tabla 6: Matriz de Impacto económico

\begin{tabular}{llllllll}
\hline Adquisición de equipos & -3 & -2 & -1 & 0 & 1 & 2 & 3
\end{tabular}

Adquisición de materiales de

$\mathrm{X}$ oficina

\section{Adquisición de equipos}

Total
$\mathrm{X}$

$-1$

2

$$
\sum=1
$$

$$
\begin{gathered}
\text { Nivel de impacto económico }=\frac{\sum}{\text { Número de Indicadores }} \\
\qquad \mathrm{NI}=\frac{1}{2}=0.5
\end{gathered}
$$

Nivel de Impacto económico $=$ Medio positivo

\section{ELABORADO POR: GRUPO DE INVESTIGACIÓN.}

La adquisición de equipos juega un papel muy importante ya que sin estos sería imposible el funcionamiento de la aplicación, la universidad ya cuenta con las máquinas y la infraestructura informática, lo único que tocaría comprar son los dispositivos biométricos de huella dactilar necesarios, por eso se le ha dado un valor de bajo negativo a este indicador, debido a que los mencionados dispositivos tienen un bajo costo. La adquisición de materiales de oficina para el proceso electoral ya no será catalogada como un egreso en la universidad ya que debido a la automatización de este proceso no se imprimirán más hojas de votación, por eso se ha valorado como medio positivo este indicador. 


\section{Impacto General.}

Tabla 7: Matriz de Impacto general.

\begin{tabular}{lllllllll}
\hline Impacto Tecnológico & -3 & -2 & -1 & 0 & 1 & 2 & 3
\end{tabular}

Impacto Ecológico

Impacto Administrativo

Impacto Económico

Total
$\mathrm{X}$

$\mathrm{X}$

$\mathrm{X}$

24

$$
\sum=6
$$

$$
\begin{gathered}
\text { Nivel de impacto general }=\frac{\sum}{\text { Número de Indicadores }} \\
\qquad \mathrm{NI}=\frac{6}{4}=1.5
\end{gathered}
$$

\section{Nivel de impacto general $=$ Medio Positivo}

\section{ELABORADO POR: GRUPO DE INVESTIGACIÓN.}

El proyecto tiene un impacto tecnológico medio positivo, con un valor de 2.3 , debido a que se agilita el proceso de escrutinio de manera eficiente, al mismo tiempo se automatiza todo el proceso de las elecciones estudiantiles. El impacto ecológico tiene un valor de 0.5 , debido a que se disminuirá en su mayor parte el uso del papel puesto que todo será digital, este impacto se valora como bajo positivo. El impacto administrativo tiene un impacto medio positivo, con un valor de 2 debido a que el nivel de organización de información mejora notablemente al ser toda automatizada. El impacto económico tiene un impacto bajo positivo, con un valor de 0.5 por agilitar el proceso del escrutinio en su totalidad y mostrar resultados después de finalizar el evento de las elecciones.

\section{Conclusiones.}

- Actualmente, el proceso de elecciones en la PUCESE es manual y tiene dificultades que se generan producto del mismo, por lo que fue necesario realizar un sistema que automatice el proceso. Además, se pudo desarrollar un sistema de votación electrónico de tipo web que cumple con los requerimientos del Tribunal Electoral 
Estudiantil de la PUCESE, para poder realizar las votaciones estudiantiles de forma automática y dinámica.

- Gracias al sistema de votación electrónico en la PUCESE se logra controlar aquellos procesos que generaban dificultades y muchos malestares al Tribunal Electoral Estudiantil, la gestión de tiempos es una parte positiva, ya que se reduce al máximo en los procedimientos de votaciones en la PUCESE, los estudiantes son beneficiados, porque no tendrán problemas de reportes sancionatorios erróneos, y se sentirán atraídos por el uso de tecnología en un proceso que carecía de ello.

\section{Referencias bibliográficas.}

Alvarez, M. A. (2010). Introducción a los lenguajes del web. 3.

Ángel, P. Y. (2013). Proyectos, Tesis y Marco Lógico (Primera ed., Vol. I). (B. Astudillo, R. Gomez, \& J. Alarcón, Edits.) Quito, Pichincha, Ecuador: Trabajo de Grado I. Recuperado el 29 de 04 de 2016

Cobo, A., Gomez, P., Perez, D., \& Rocha, R. (2005). PHP y MySQL Tecnologías para el desarrollo de aplicaciones web. España: Rústica-Hilo. doi:84-7978-706-6

Microsoft SQL Server. (2015). SQL Server and Microsoft Azure. Recuperado el 20 de 01 de 2018, de Microsoft: http://download.microsoft.com/download/1/8/3/183CE8F8EFC0-4C60-97B2-

5CC5201517B8/SQL_Server_Mission_Critical_Performance_White_Paper_esES.pdf

Secugen Corporation. (12 de 04 de 2012). Recuperado el 07 de 04 de 2016, de secugen: http://www.secugen.com/products/php.htm

Tuesta Soldevilla, F. (2004). El voto electrónico. Recuperado el 13 de 01 de 2018, de congreso.org:

http://www2.congreso.gob.pe/sicr/cendocbib/con2_uibd.nsf/3A3956525DE46E9C0 52575A200649D08/\$FILE/1votito.pdf

Velázquez Trujillo, H. (2010). El voto electrónico en las elecciones de alcaldes en Venezuela.

Recuperado el 5 de 01 de 2018, de http://www2.congreso.gob.pe: http://www2.congreso.gob.pe/sicr/cendocbib/con4_uibd.nsf/8C23E1544ADE5ABC 05257B570078F33D/\$FILE/67614336007.pdf 
Yépez, M. Á. (2013). PROYECTOS, TESIS Y MARCO LÓGICO. (B. Astudillo, R. Gomez, \& R. Alarcón, Edits.) Quito, Pichincha, Ecuador. Recuperado el 05 de 12 de 2017

Zissis, D., \& Lekkas, D. (2011). Securing e-Government and e-voting.

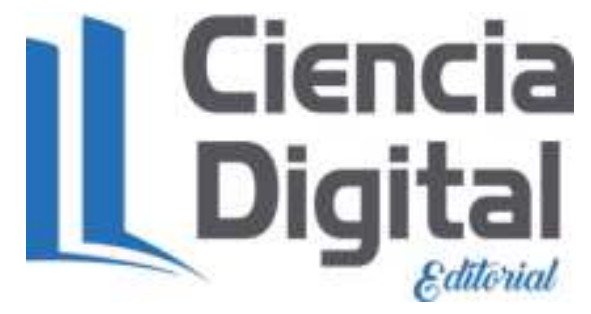




\section{Para citar el artículo indexado.}

Macías R., Mendoza A., Puyol J. \& Guamán R. (2018). Desarrollo de un sistema de votación electrónico con mecanismo biométrico de autentificación. Revista electrónica Ciencia Digital 2(1), 246-262. Recuperado desde:

http://www.cienciadigital.org/revistascienciadigital2/index.php/CienciaDigital/article/view/ $17 / 17$

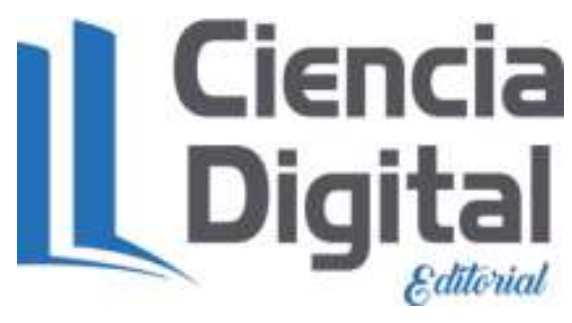

El artículo que se publica es de exclusiva responsabilidad de los autores y no necesariamente reflejan el pensamiento de la Revista Ciencia Digital.

El articulo queda en propiedad de la revista y, por tanto, su publicación parcial y/o total en otro medio tiene que ser autorizado por el director de la Revista Ciencia Digital.
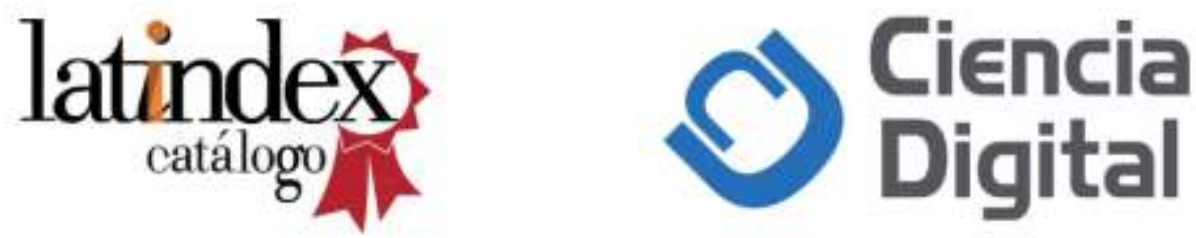